\title{
SAFETY IN ANAESTHETIC PRACTICE
}

\author{
Kalyani Nihalsinghe* \\ Consultant Anaesthetist, Sri Lanka \\ *Corresponding author E-mail: knihalsinghe@yahoo.com
}

Key words: Global awareness in safety, Protocols, Guidelines, Equipment checks, Quality assurance, monitoring standards, Education

\section{Introduction}

Anaesthesiology is acknowledged as the leading medical specialty which addresses patient safety. Anaesthesia has undergone substantial development and sophistication from its early beginnings; it is now safer than ever owing to many different types of improvement solutions and strategies implemented. These include new technologies, standards and guidelines related to human factors and system issues ${ }^{1}$

\section{Professional concern over safety}

There was concern among professional bodies that patient safety was imperfect and that like any other medical problem, patient safety should be studied and interventions planned to better outcomes. The formation in 1985 of a multi disciplinary Anaesthesia Safety Foundation in the US was a land mark in institutionalization and legitimization of patient safety as a topic of professional concern. The Foundation has advocated a single standard of safety for surgical and anaesthetic procedures, whether these take place in hospital or in outpatient unit ${ }^{1}$. The Australian Safety Foundation set up in 1988 follows similar policies. The Association of Anaesthetists of Great Britain and Ireland as well as Australian New Zealand College have published extensive guidelines for promoting safety which are updated regularly. Recently, Sri Lanka College of Anaesthesiology has set up guide lines for safe practice.

\section{Standards and guide lines}

The practice of modern anaesthesia has become complex, technological, and has expanded to include intensive care. The need for the anaesthetic provider to develop a high degree of competence is essential. Such a provider should have:

1. High level of knowledge and sound judgment.

2. Respond quickly and accurately to clinical situations.

3. Able to sustain long periods of vigilance.

\section{Minimum requirements for a safe practice $^{2}$.}

1. Continuous presence of a qualified anaesthetist with a trained assistant.

2. Reliable, regularly serviced equipment.

3. A protocol for detail systems check of anaesthetic and monitoring equipment.

4. System of recording and auditing the process.

Technological solutions which are implemented in modern practice are ${ }^{1}$.

1. Real time monitoring of patients electronically and clinically.

2. Use of engineered safety devices e.g non interchangeable gas cylinder yokes.

3. New technologies for managing patients airways e.g. availability of airway manipulation equipment like fibreoptic laryngoscopes and intubation devices.

\section{System issues}

It is necessary to follow the guide lines laid out by the institutions for checking equipment. A pre session check of all anaesthetic and monitoring devices must be done and recorded. New equipment should be demonstrated to all personal involved in their usage before putting them into regular use as a ‘quick' run can end up in disaster. 
The consultant or the senior anaesthetist must take the responsibility to arrange time allocations for the demonstrations. Updating of check lists is mandatory when new equipment is installed as there is introduction of microprocessor technology and change in equipment design.

\section{Human factors leading to accidents - Fatigue $^{3}$.}

The most overlooked or neglected human factor is fatigue among the anaesthetist and associated staff. Fatigue has proven to impair vigilance and accuracy of response. It also decreases performance of motor and cognitive function, impairs judgment and results in delay, and inadequate responses to clinical changes. Fatigue will also result in poor communication and inadequate record keeping. The health care employer must implement strategies to provide safe working schedules to avoid risks to patients and staff. For shift work, forward rotating shift e.g. morning > evening> nights have been proven to be the best as it cause least disturbance to normal sleep patterns. The Department of Health must have a national code of practice for hours of shift work and rosters.

Protocol for transfer of responsibility ${ }^{3}$. A system of transferring responsibility from shift to shift must be strictly followed. This is especially important as very often mishaps occur during this time. The primary anaesthetist when handing over a patient should be satisfied with the competence of the relieving anaesthetist and the handover must be done when the clinical status of the patient is stable. There must also be a clear understanding that the relieving anaesthetist is willing to accept the responsibility for the case.

\section{Infection risks to patients}

During invasive procedures e.g. vascular cannulations, intrathecal injections, strict aseptic precautions must be followed. The anaesthetic and airway management apparatus carry a potential risk of infection by repeated use and should be properly sterilized between cases. Drugs and syringes must be placed on a sterile trolley to prevent contamination.
Infection risks to health care workers can be avoided by following a simple protocol e.g hand washing, wearing gloves and disposing sharps safely.

\section{Quality Assurance programmes ${ }^{4}$.}

Quality assurance is a process of on going review of patient care and such programmes are necessary to improve patient care, to identify and highlight underlying characteristics of mishaps. The objective is to ensure high standards through regular assessments. The results must be reported for evaluation for necessary action to be taken.

\section{Some of the quality assurance programmes which give valuable information are}

1. Regular Mortality Morbidity meetings

2. Statistics of clinical work.

3. Incident reporting.

4. Management review of random topics

5. Performance of the Department as a whole e.g. staffing, numbers, seniority, duties etc

6. Expenditure for the department for teaching, research \& for equipment

7. Patient management- preoperative assessment, monitoring techniques, record keeping.

8. Post anaesthetic management-criteria for admission to Recovery Unit, pain relief etc

9. Patient outcome assessment by the following clinical indicators:

o Percentage of patients for elective surgery having a preoperative visit

o Mortality within 24 hours of administration of anaesthetic

o Failure to be discharged from recovery 2hours after an anaesthetic

o Unplanned admission to ICU within 24 hours after an anaesthetic

o Injuries attributed to anaesthesia

\section{Critical Incident (CI)}

$\mathrm{CI}$ is an occurrence if not discovered or corrected on time can lead to a substantive negative outcome. It can range from increased hospital stay, death or permanent disability. The incident could be clearly preventable 
CI studies were first introduced by Flanagan in 1954 and developed to reduce loss of military pilots and aircrafts in training. Cooper in 1978 modified and adopted this method for anaesthesia. Analysis of Critical incidents reveal the root cause of accidents, and they are categorized into the following ${ }^{6}$.

- Human error -due to inadequate communication / haste / distraction / fatigue / lack of knowledge.

- Equipment failure-breathing circuit disconnection/ventilator malfunction/gas flow control errors etc

- Airway problems

- Pharmacological (wrong drug )

Human error is a pervasive and normal part of every day life and in anesthetic practice errors lead to incidents and accidents.

Human error can be active or latent. Studies have revealed that active errors result from the following lapses.

- Knowledge based - lack of knowledge regarding the procedure

- Rule based - If protocols or algorithms were not followed

- Skill based - slips and lapses as a result of lack of supervision

- Technical errors- unfamiliar with equipment, failure to check

Latent errors lead to substantial problems in anaesthesia and require systematic, continuous attention to reduce and eliminate. These include the working environment, physiological state, equipment condition and reliability, work practices, personal training, social and cultural factors.

Following studies highlighted the category of error

1. A study of human factors ${ }^{7}$ in US. The number of preventable incidents reported were 359.of which human error was $82 \%$ \& equipment failure $14 \%$

2. ASA closed claims project $^{5}$ analysed 3791 cases retrospectively for a 10 year period and found the majority was due to equipment failure

3. Australian incident monitoring study (AIMS) $)^{8}$ reported 2000 incidents of which equipment failure in general was 177 (9\%). Of which anaesthesia delivery system failure was $107(60 \%)$

4. Drug related incidents were analysed In 896 from the AIMS database. 452(50.4\%) syringe \& drug preparation error. $169-(18.9 \%)$ syringe swaps-drug correctly labeled but given in error. 187-(20.8\%) selection of wrong ampoule or drug labeling error. Most common drugs were neuromuscular blockers and opioids ${ }^{10}$.

Ten frequently occurring incidents revealed in data analysis ${ }^{7}$.

1. Breathing circuit disconnection

2. Breathing circuit connection error

3. Inadvertant gas flow change.

4. Syringe swap

5. Gas supply problem.

6. IV apparatus disconnection.

7. Laryngoscope malfunction

8. Premature extubation

9. Hypovolemia

10. Tracheal airway device position change

Since accidents cannot be completely abolished data analysis is a very useful guide to implement strategies to prevent and minimize their occurrence. It has been well established that in order to improve patient safety that a set of minimum monitoring standards must be implemented. The first set of standards of basic monitoring was developed by the Harvard hospitals and later adopted by the American Society of Anaesthetists in 1986 and the recommendations were made as National policy ${ }^{\mathbf{1}}$. Later in 1989 the International Task Force applied these recommendations to improve quality and safety which were endorsed by the World Federation of the Society of Anaesthesiologists. These Monitoring Standards are simple and easy 
to apply and should be used in all patients subjected to an anaesthetic ${ }^{9}$.

1 Oxygenation-Observation of patient, Use of Oxygen analyzer, Pulse Oximeter

2. Circulation - Palpation of pulse, Auscultation of heart sounds Continuous ECG display, Heart rate and Blood pressure every 5 minutes, temperature, evaluation of circulation etc

3. Ventilation - Observation of patient, reservoir bag, auscultation of breath sounds and Capnography.

Present day monitors have improved remarkably and most monitors can detect and prevent mishaps if used correctly. Advantage of multiple monitors is that they can cross check each other. In the event of monitor malfunction the patient should be checked first as such the anaesthetist must be physically available at all times.

The practice of anaesthesia has developed into a specialty focused mainly on safety as a result of better monitoring, safer medications and with advances in knowledge and training. But the various safety advances require a long term commitment to achieve their full promise

\section{References}

1. David M Gaba; as a model for patient safety in health care Anaesthesiology BMJ2000;320;785788,

2. Recommendations for standards of monitoring during anaesthesia and recovery, AAGBI $4^{\text {th }}$ edition 2007

3. Australian \& New Zealand College of Anaesthetists; Review, PS43 (2001) PS10(2004)

4. The Hong Kong College of Anaesthesiologists; Guide lines on quality assurance, p7; 2002

5. James Eisenkraft MD: CJA51:R7 (2004), Hazards of Anaesthesia Delivery Systems.

6. 6.Runciman WB,Sellen A,Webb RK Williamson JA,Currie M,Morgan C,Russel $\mathrm{WJ}$; The Australian Incident monitoring study, errors, incidents and accidents in anaesthetic practice Anaesthesia \& Intensive care Oct;21(5);506-19)

7. 1.Cooper JB, Preventable anaesthesia mishaps and study of human factors Anaesthesiology, 1978 Dec:49(6)399-406.

8. RK Webb, Currie M, Morgan CA, Makey, Russel WJ, Runciman W,

The Australian incident monitoring study:an analysis of 2000 Incident reports Anaesthesia and Intensive Care, 1993 October; 21(5):520-8

9. Indian J anaesthesia, 2002;46(4):244-255 , Editorial

10. A.Abeysekera, J Bergman, MT Kluger,TG Short Medication error in anaesthetic practice. Review of 896 reports from AIMS study database Anaesthesia vol 60 issue 3, 220-227 May 2005 